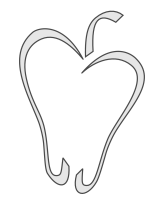

ISSN 1852-4249

\title{
Commentary on "Anisotropic finite-size scaling of an elastic string at the depinning threshold in a random-periodic medium"
}

\author{
Andrei A. Fedorenko ${ }^{1 *}$
}

In their paper [1] S. Bustinagorry and A.B. Kolton study the finite-size scaling properties of an elastic string driven in a disordered medium at the depinning transition. The zero-temperature dynamics of the model is given by the equation of motion for the displacement field $u(z, t)$ :

$$
\gamma \partial_{t} u(z, t)=c \partial_{z}^{2} u(z, t)+F_{p}(u, z)+F,
$$

where $\gamma$ is the friction, $c$ the elasticity, and $\mathrm{F}$ the driving force. $F_{p}$ is the Gaussian random force due to disorder with zero mean and variance

$$
\overline{F_{p}(u, z) F_{p}\left(u^{\prime}, z^{\prime}\right)}=\Delta\left(u-u^{\prime}\right) \delta\left(z-z^{\prime}\right) .
$$

The model of an elastic object in a disordered medium can be used to describe diverse physical systems. They can be cast into two classes: (i) periodic systems, such as charge density waves (CDW) in solids and vortex lattice in disordered superconductors; (ii) interfaces such as domain walls in magnets. $\Delta(u)$ is a periodic function for the random periodic systems and a short range function for the interfaces. Due to the competition between elasticity and disorder, these systems exhibit a rich glassy behavior and a non-trivial response to external perturbations. In particular, a driving force $F$ exceeding a certain threshold value $F_{c}$ is required

*E-mail: andrey.fedorenko@ens-lyon.fr

1 CNRS-Laboratoire de Physique de l'Ecole Normale Supérieure de Lyon, 46, Allée d'Italie, 69007 Lyon, France. to set the elastic object into steady motion. To some extent, the depinning transition shares many features with critical phenomena where the velocity $v$ plays the role of the order parameter. For instance, the correlation length defined through the velocity-velocity correlation function diverges when approaching the transition from above as $\xi \sim\left(F-F_{c}\right)^{-\nu}$. However, in comparison with the ordinary critical phenomena, the problem of disordered elastic systems is notably difficult due to the so-called dimensional reduction. The latter takes place also in random field spin systems and states that a $d$-dimensional disordered system at zero temperature is equivalent to all orders in perturbation theory to a pure system in $d-2$ dimensions at finite temperature. It turns out that the metastability renders the zero-temperature perturbation theory useless: it breaks down on scales larger than the socalled Larkin length. The peculiarity of the problem is that for $d<4$ there is an infinite set of relevant operators, which can be parameterized by the function $\Delta(u)=-R^{\prime \prime}(u)$. The corresponding functional renormalization group (FRG) equation for the renormalized disorder correlator has two fixed points: the random field fixed point (RFFP) which describes the depinning transition for the interfaces and the random periodic fixed point (RPFP) which describes the random periodic systems. The running (renormalized) disorder correlator becomes a non-analytic function beyond the Larkin scale. The appearance of a non-analyticity in the form of a cusp at the origin is related to metastability, and 
Papers in Physics, vol. 2, ARt. 020009 (2010) / A. A. Fedorenko

nicely accounts for the generation of a threshold force at the depinning transition: $\overline{F_{c}} \sim-\Delta^{* \prime}\left(0^{+}\right)$. Note, that the latter is zero in the bare (analytic) theory. Thus, FRG provides a way to analytically compute the threshold force which turns out to be nonuniversal (similar to the critical temperature in critical phenomena) and the critical exponents which depend only on the universality class. In particular, one can compute the roughness exponent which gives the scaling of the average width of the interface with its size: $\overline{w^{2}} \sim L^{2 \zeta}$. The observables computed using FRG in the thermodynamic limit are already averaged over different disorder realizations. The picture for finite systems is more involved.

In numerical simulations, one usually considers a finite system of linear size $L$ moving in a box of size $M$ with periodic boundary conditions. Then, depending on how one performs the finite-size scaling analysis, one can approach different scaling regimes. In the limit of $L \rightarrow \infty$ at fixed $M$ one approaches the scaling behavior of a random periodic system. In the opposite limit of $M \rightarrow \infty$ at fixed $L$, the system falls in one of the universality classes of a particle moving in a random landscape described by some particle fixed point (PFP) [2]. The random field universality class describing the scaling properties of a pinned interface corresponds to the limit of both $L, M \rightarrow \infty$. However, one has to specify the precise way of taking this limit in order to separate the basin of attraction of the RFFP from those of the RPFP and the PFP. Studying the crossover between these three universality classes is the main aim of the paper [1].

The key parameter is $k=M / L^{\zeta_{\text {dep }}}$ where $\zeta_{\text {dep }}$ is the roughness exponent of the interface (a string) at the depinning transition. It has been found in [1] that $\overline{w^{2}} L^{-2 \zeta_{\text {dep }}}$ is a universal function of $k, G(k)$, which has a minimum at $k^{*}<1$. For $k \ll k^{*}$, one reveals that the periodicity is important and the system is described by the RPFP. In this case, there are two relevant contributions to the displacement correlation function: the universal logarithmic growth with zero roughness exponent and the Larkin type growth due to instability of RPFP which gives the roughness exponent $\zeta_{\mathrm{L}}=(4-d) / 2$.

For $k \approx k^{*}$, one expects that the system approaches the RFFP universality class in the limit $L \rightarrow \infty$. The function $G(k)$ for $0<k<k^{*}$ describes the crossover between the RPRF and the
RFFP. The main result of the paper is that in order to verify the predictions of FRG and numerically study the properties of the RFFP universality class, one has to keep $k=k^{*}$. In the case of deviations from $k=k^{*}$, the results are modified by the crossover either to the RPFP or to the PFP.

It has been found that $G(k)$ increases slower than any power-law for $k>k^{*}$. In this regime, the system is expected to show crossover to the PFP [2]. The properties of the critical configuration are determined by the extreme value statistics. For very large $k$, one can split the system in $k$ almost uncorrelated regions with their own subcritical configurations. The true critical configuration corresponds to the subcritical configuration with the maximal critical force. As a result, in the limit $k \rightarrow \infty$, the distribution of the threshold force exhibits crossover from the Gaussian distribution to the Gumbel distribution. The average critical force increases as $F_{c} \sim \log k$ since the critical force can be regarded as the maximum among $k$ different subcritical forces. The authors argue that the increase in the critical force might be correlated with the slow increase of roughness. They suggest that the larger critical force can be achieved either by accidental extended defects or by rare uncorrelated strong pinning forces. They argue that the second scenario is more plausible since the presence of accidental correlation should decrease roughness. However, as it was shown in [3], the presence of correlated disorder and extended defects also enhances roughness. Therefore, one can expect the simultaneous enhancement of the roughness and the critical force.

The similar crossover effects have been observed by the authors in the behavior of the structure factor and the interface width distribution.

Summarizing, the authors numerically observe that the average width and its probability distribution depend, in a universal way, on the parameter $k$ that is encoded in the universal function $G(k)$. The parameter $k$ is decisive regarding the universality class which will be approached in the thermodynamic limit or probed by the finite size scaling analysis. Knowing the function $G(k)$, and especially the position of its minima $k^{*}$, is required for the correct analysis of the numerical simulations. Analytical calculation of $G(k)$ using, for example, FRG remains, however, an open challenging problem. 
Papers in Physics, vol. 2, ART. 020009 (2010) / A. A. Fedorenko

[1] S Bustingorry, A B Kolton, Anisotropic finitesize scaling of an elastic string at the depinning threshold in a random-periodic medium, Pap. Phys. 2, 020008 (2010).
[2] P Le Doussal, K J Wiese, Driven particle in a random landscape: disorder correlator, avalanche distribution and extreme value statistics of records, Phys. Rev. E 79, 051105 (2009).

[3] A A Fedorenko, P Le Doussal, K J Wiese, Statics and dynamics of elastic manifolds in media with long-range correlated disorder, Phys. Rev. E 74, 061109 (2006). 